\title{
La FASP médicale et ses marges : textes de référence, prototextes et textes périphériques
}

Jean-Pierre Charpy

\section{OpenEdition}

\section{Journals}

Édition électronique

URL : http://journals.openedition.org/asp/795

DOI : $10.4000 /$ asp.795

ISBN : 978-2-8218-0400-5

ISSN : 2108-6354

Éditeur

Groupe d'étude et de recherche en anglais de spécialité

Édition imprimée

Date de publication : 1 janvier 2005

Pagination : 83-101

ISSN : 1246-8185

\section{Référence électronique}

Jean-Pierre Charpy, «La FASP médicale et ses marges : textes de référence, prototextes et textes périphériques », ASp [En ligne], 47-48 | 2005, mis en ligne le 16 février 2010, consulté le 06 mai 2019. URL : http://journals.openedition.org/asp/795 ; DOI : 10.4000/asp.795

Ce document a été généré automatiquement le 6 mai 2019

Tous droits réservés 


\section{La FASP médicale et ses marges: textes de référence, prototextes et textes périphériques}

Jean-Pierre Charpy

\section{Introduction}

1 Parmi les genres rattachés à la notion de fiction à substrat professionnel (FASP), la FASP médicale a fait l'objet de divers travaux de recherche ${ }^{1}$. Ces travaux ont abouti au repérage d'un groupe restreint d'auteurs américains (R. Cook, M. Palmer et T. Gerritsen), tous représentatifs de ce genre spécialisé, et à la constitution d'un noyau dur de textes contemporains caractérisés par leur succès commercial, un tissu narratif et linguistique à dominante professionnelle et des auteurs appartenant ou ayant appartenu au milieu médical.

2 Les textes fondateurs comportent les éléments constitutifs emblématiques de la FASP et à ce titre, il est légitime de penser qu'ils forment naturellement le cœur de la FASP médicale. Toutefois, ce genre spécialisé, bien qu'encore jeune, semble être entré dans un processus évolutif sensible. Il convient donc de vérifier si des romans affiliés - de près ou de loin - à la FASP médicale, contribuent à cette évolution récente.

3 Soucieux d'élargir notre recherche à des textes situés aux frontières diffuses de la FASP médicale, nous proposons d'étudier les productions, a priori marginales, d'auteurs dont les romans ne répondent pas totalement aux normes précédemment définies.

4 Ainsi, F. G. Slaughter, à partir des années 1940, et M. Crichton, dans les années 1960, ontils écrit des romans à forte influence médicale. Ces textes semblent être hors normes du fait de leur publication anachronique quant à la définition du genre de la FASP et, dans le cas de F. G. Slaughter, en raison de l'absence totale de suspense. En dépit de ces carences génériques, ne doivent-ils pas être considérés comme des prototextes de la FASP médicale ayant favorisé l'éclosion du genre? 
De la même manière J. Connelly, ambulancier de profession et auteur atypique, a écrit un roman à dominante médicale Bringing out the Dead sans avoir recours aux ressorts de l'intrigue des "medical thrillers» classiques. D'autres écrivains, comme G. Braver, universitaire de formation, et $\mathrm{K}$. Reichs, issue du milieu de l'anthropologie médico-légale, n'appartiennent pas au sens strict au milieu professionnel de la médecine.

Toutefois, le substrat professionnel à influence biomédicale ou médico-légale qui soustend leurs romans à suspense présente certaines similitudes troublantes avec celui des textes fondateurs. La question se pose donc de savoir si ces textes périphériques qui, en apparence, gravitent dans une zone frontière large de l'ensemble de la FASP médicale, doivent être classés aux confins du genre ou y être intégrés en tant que productions hybrides.

Plusieurs indices laissent à penser que la FASP médicale connaît actuellement un processus d'évolution propre à la variation des genres ; à savoir, l'intégration progressive d'éléments provenant de genres littéraires classiques (roman policier, roman de sciencefiction, roman d'épouvante) et la fusion d'éléments de genres spécialisés connexes opérée par un auteur emblématique de la FASP médicale, T. Gerritsen, dans son roman récent The Sinner.

8 Dans la mesure où, désormais, la distinction entre le cœur et les marges du genre spécialisé est parfois difficile à percevoir, nous postulons que, si le substrat professionnel est un trait constant fondamental du domaine de la FASP médicale, il existe des zones floues du domaine où, grâce à un processus de diffusion sélective qui s'apparente à l'osmose, l'apport d'éléments non emblématiques donne une impulsion nouvelle au genre en lui apportant les variations nécessaires à son évolution.

\section{De la fiction à substrat professionnel}

\subsection{FASP et substrat professionnel}

9 La notion de fiction à substrat professionnel et le genre de la FASP ont été définis et clarifiés par M. Petit dans divers articles fondateurs (1999, 2000, 2004). Par nature, les œuvres de création (romanesques, cinématographiques, télévisuelles,...) appartenant au genre de la FASP sont étroitement liées au mode d'expression et à la culture emblématique de divers milieux professionnels.

10 Le genre général de la FASP, en tant qu'objet littéraire, se décline en genres particuliers (FASP juridique, FASP médicale, FASP militaire) qui constituent des groupes génériques spécialisés. Il s'agit de productions émanant de milieux professionnels dont l'intérêt épistémologique réside dans le fait qu'ils dépassent la simple représentation qu'ils en donnent :

C'est ce milieu professionnel « réel », la réalité sociale préexistante, qui constitue à proprement parler le substrat, et non pas le milieu professionnel représenté dans la fiction. (Petit $2004: 14$ )

11 La forte prégnance du réel dans les productions de la FASP peut être jugée à l'aune de l'expérience professionnelle, passée ou présente, des auteurs ou des créateurs, mais aussi à leur impact sur les communautés professionnelles ciblées. Comme l'indique M. Petit, il faut faire la distinction entre la notion de «substrat professionnel", liée à la connaissance interne, donc intime, du milieu professionnel, et celle d'«adstrat 
professionnel » (1999: 67) ou «adstrat spécialisé » (2004: 20), liée à une connaissance externe résultant de la fréquentation du milieu professionnel.

Cet extrait du paratexte de The Bernini Bust de I. Pears (2001: i), quiillustre The Raphael Affair,résume bien le statut d'auteurs de fiction proches du milieu professionnel, mais n'en ayant pas nécessairement une connaissance intime: "Presents a world the author knows well in the satisfying way Margaret Truman and Dick Francis set their mysteries in milieus they know » (the Associated Press).

13 L'étalon-or permettant d'identifier les productions avérées du genre de la FASP repose sur plusieurs critères stricts, recensés par M. Petit (2004:5). Il classe dans le domaine de la fiction à substrat professionnel les productions contemporaines à visée commerciale, conçues par des auteurs ou des créateurs appartenant ou ayant appartenu au milieu professionnel. Ces romans ont (de préférence, mais pas exclusivement) une intrigue à rebondissements. Leur contenu narratif et discursif est à dominante professionnelle.

Ce recours à la norme permet de définir clairement les frontières du domaine et les limites du genre. Cependant, d'autres chercheurs, comme S. Isani, pensent qu'il est souhaitable d'aller au-delà de l'approche normative pour tenter de cerner les évolutions et les variations du genre :

The core conventions of the genre having been established, we propose to move beyond the "classics" and explore other possible forms of the FASP on the basis of the hypothesis that genre is not formulaic and fossilised dogma but a shifting and permeable set of dynamics in constant negotiation and evolution. (2004:27)

Nous proposons maintenant d'explorer le domaine quelque peu diffus de la FASP médicale et d'y chercher les indices qui permettront de savoir si ce genre spécialisé est figé dans la normalité ou s'il se transforme progressivement.

\subsection{FASP et FASP médicale}

e territoire largement inexploité de la FASP médicale est en cours d'exploration. Dans deux articles précédents, nous avons tenté de dessiner la topographie de cette vaste parcelle de la FASP et de baliser ses relations étroites avec le milieu professionnel de la médecine ${ }^{2}$.

17 Nous avons abordé exclusivement les productions de la FASP romanesque ${ }^{3}$, communément appelées «medical thrillers", en raison de leur succès auprès du grand public, de leur écho favorable auprès de la profession et de leur portée pédagogique. Comme le fait remarquer G. Diaz-Santos, ces textes présentent un intérêt réel en complément de sources primaires telles que l'article de recherche ou de synthèse :

One advantage of a literary text over a specialist one - at least in a language teaching context - is precisely its potential to engage readers' imaginative faculties.

(2000: 229)

18 Les romans appartenant à ce genre spécialisé, qui associent thèmes médicaux et suspense, connaissent un succès populaire depuis une trentaine d'années. Toutefois, il convient de noter que, dans le cadre de la transmutation des genres, ces romans à suspense à dominante médicale portent l'empreinte générique de caractéristiques littéraires anciennes identifiables dans des romans gothiques comme The Mysteries of Udolpho (Radcliffe 1794) ou The Monk (Lewis 1796), mais surtout dans The Woman in White (Collins 1860) et dans certaines nouvelles d'E. A. Poe écrites au XIX siècle. 
19 Pour reprendre la terminologie utilisée par M. Petit, nous parlerons désormais de genre spécialisé pour désigner la FASP médicale dans sa globalité, et non plus de sous-groupe générique comme nous l'avions fait dans les articles mentionnés ci-dessus. En effet, dans un souci de clarté, nous souhaitons privilégier le cadre formel générique de la FASP médicale, qui répond à des critères de stabilité et de reconnaissance identifiés précédemment : fiction commerciale contemporaine, intrigue haletante se déroulant en partie dans un contexte médical, discours à dominante médicale, auteurs issus du milieu professionnel de la médecine.

Certes, il est toujours possible de subdiviser un genre en sous-groupes génériques. L'expression «forensic thrillers» désigne fréquemment les productions commerciales d'auteurs comme K. Reichs et P. Cornwell. Les romans récents de T. Gerritsen sont parfois classés dans la catégorie "gothic medical thrillers».

21 Comme en atteste cet extrait de Fever Cell, le roman de L. Goldberg pourrait fort bien être répertorié dans un sous-groupe baptisé pour l'occasion " gore medical thrillers »:

They had been at Kathy's bedside, talking with her, when the young nurse started to retch and heave. At first nothing came up. Then all hell broke loose. Black blood suddenly gushed out of her mouth and nose and rectum. It came out in torrents and flooded onto the floor, covering everything in black gook. Kathy Wells bled out in less than two minutes, and died with her eyes wide open. (2003:171)

Il semble donc que, pour la cohérence de notre propos, le terme de genre spécialisé appliqué aux productions de la FASP médicale soit adéquat car les principaux traits du roman à suspense (intrigue complexe, recours systématique aux émotions fortes, poursuites échevelées,...) y sont entrecroisés avec les éléments constitutifs de l'environnement professionnel médical.

23 Ce substrat professionnel crédible produit par des membres ou d'ex-membres de la communauté médicale comporte généralement un discours spécialisé évitant tout jargon car il est destiné en priorité au grand public. À ce sujet, voici quelques conseils prodigués aux futurs auteurs de medical thrillers par le médecin-romancier G. Birken lors d'un atelier d'écriture intitulé « Medical Fiction Writing ":

"The overdose syndrome" - adding the right amount of medicine to your medical thriller's recipe. Conveying medical information - talking to your reader in the same way you talk to your patients. (Birken 2005)

L'adaptation nécessaire du discours spécialisé dans les romans à suspense à dominante médicale ${ }^{4}$ montre que la FASP médicale offre une représentation, parfois même une vulgarisation, des traits caractéristiques du monde de la médecine. Pour reprendre la terminologie de M.Bakhtine, le genre spécialisé constitue une sphère d'échange intéressante entre genre de discours premier et genre de discours second. Toutefois, les medical thrillers ont également un fort impact sur le milieu professionnel lui-même.

Ainsi, l'organisation d'ateliers d'écriture par des professionnels pour des professionnels nous incite à penser que de nombreux membres de la communauté médicale sont sensibles à la rédaction de ces romans populaires. Ce commentaire au sujet de $\mathrm{G}$. Birken est révélateur: «Gary will explain how he is able to continue his busy and successful medical practice as a pediatric surgeon and still find the time to write medical thrillers. » (Birken 2005).

En outre, plusieurs auteurs à succès continuent d'exercer leur activité professionnelle d'origine, tout en écrivant. D. M. Shobin, auteur de six « medical suspense novels ", pratique la médecine vingt-cinq à trente heures par semaine. De même, P. Clement a écrit sept 
romans à suspense à dominante médicale tout en travaillant à plein temps au service des urgences.

B. Pomidor, quant à lui, a pratiqué la médecine en tant que médecin de famille avant de devenir enseignant et écrivain. Il a déjà écrit cinq « mystery novels » qui mettent en scène un couple de professionnels de la santé, Plato et Calista Marley. Il est intéressant de noter que B. Pomidor utilise les romans de T. Gerritsen dans le cadre de la formation initiale d'étudiants en médecine: "Also, my novels were used in a Medical School class in fiction in Ohio. The teacher's name was Bill Pomidor, M.D. $»^{5}$.

D'autres éléments objectifs tendent à montrer que la FASP médicale n'est pas une simple mise en scène de la communauté médicale, mais en est partie intégrante. Le site officiel de laBritish Medical Association (BMA) comporte une rubrique intitulée « Fiction Writers with Medical Qualifications »; elle fournit des liens avec des sites généralistes sur la fiction médicale ainsi qu'une liste d'une centaine de professionnels de la santé ayant (ou ayant eu) une carrière ou des velléités littéraires. Bien qu'ayant eu une formation médicale, certains auteurs répertoriés, comme T. Smollett, Sir Arthur Conan Doyle et W. Somerset Maugham, n'ont pas produit de romans pouvant être affiliés au genre «medical fiction ». En revanche, A.J.Cronin a fréquemment introduit des thèmes médicaux dans sa production littéraire ${ }^{6}$.

Une recherche par mots-clés («medical thriller») sur le site officiel du Journal of the American Association (JAMA) permet de constater qu'une bonne dizaine de medical thrillers - dont Miracle Cure (1998) de M. Palmer - ont fait l'objet d'une critique ou d'un commentaire dans la rubrique «Books, Journals, New Media ».

Tous ces signes convergents indiquent que le substrat professionnel qui étaye les romans de la FASP médicale est intimement lié à la culture de la communauté médicale. Il convient désormais de délimiter les contours de ce genre spécialisé de la FASP.

\section{Au cœur de la FASP médicale}

Après avoir rappelé les grandes lignes du cadre théorique et épistémologique de la fiction à substrat professionnel, nous allons désormais tenter de pénétrer au cœur de la FASP médicale en répertoriant quelques textes de référence et les principaux auteurs qui ont contribué à la naissance, la croissance et la pérennité de ce genre spécialisé.

Les romans de la FASP médicale reposent essentiellement sur un modèle conventionnel quelque peu rigide. Ce commentaire de G. Scott, extrait d'un article en ligne de la revue spécialisée Physician's Weekly, est une description plutôt étriquée de la formule de base utilisée :

Medical thrillers are formulaic. Writers take an aspect of medicine and make it frightening. Robotics, resistant organisms, genetic manipulation, and organ transplantation have been used to effect. The protagonist is almost always a sympathetic physician - often a woman - whose story involves romantic relationships, a threatened career, and critical choices involving medical ethics. (2000)

Dans le souci de dépasser les formules et les clichés fréquemment associés à ces productions commerciales, nous proposons d'utiliser les critères objectifs de caractérisation de la FASP romanesque établis par $\mathrm{M}$. Petit ${ }^{7}$ afin d'évaluer leur appartenance au genre ainsi que la pertinence de leur positionnement au sein du genre, tout en restant attentif à de potentielles variations. 


\subsection{Les textes fondateurs}

Le domaine de la FASP médicale peut être défini comme un ensemble générique spécialisé diffus formé, en son centre, de productions ayant toutes les caractéristiques emblématiques du genre et dans une zone périphérique large, de productions atypiques comportant certaines des caractéristiques génériques. La plupart des medical thrillers écrits par R. Cook, M. Palmer et T. Gerritsen ${ }^{8}$ se situent au cœur de la FASP médicale car ces romans répondent à l'ensemble des critères emblématiques. À ce titre, ils peuvent être considérés comme les textes fondateurs du genre spécialisé.

Le premier docteur (devenu écrivain) à en avoir posé les fondations est indéniablement R. Cook avec Coma (1977), suivi de près par son collègue M. Palmer, auteur de The Sisterhood (1982). Ceci est confirmé par J. Harkavy, journaliste travaillant pour The Associated Press :

Palmer, a former internist who went on to become an emergency room doctor, was inspired by the success of Robin Cook, the ophthalmologist whose 1977 best-seller, Coma, is credited with creating the medical thriller genre. Palmer knew Cook when they were undergraduates at Wesleyan; they subsequently trained together at Massachusetts General Hospital. (2002)

J. Harkavy insiste sur le caractère commercial de ces romans contemporains ( 1977 bestseller »), tout en soulignant la qualification professionnelle des auteurs. Il s'agit bien là de traits définitoires essentiels du genre. Nous postulons donc qu'à partir des années soixante-dix, le genre spécialisé de la FASP médicale a vu le jour en Nouvelle-Angleterre grâce aux pères fondateurs, R. Cook et M. Palmer. Il a pris son essor à partir des années quatre-vingt-dix avec le succès croissant des medical thrillers écrits par les auteurs de la première génération, relayés par des auteurs de la deuxième génération comme T. Gerritsen.

7 Le critère du roman commercial contemporain étant avéré pour ces auteurs, il convient désormais d'aborder celui de leur crédibilité professionnelle et de leur représentativité dans la communauté médicale. Parmi les trois romanciers cités précédemment, seul M. Palmer continue à exercer dans le milieu de la santé, parallèlement à ses activités littéraires.

Après avoir été urgentiste, ce qui lui a permis de décrire avec authenticité et vivacité les services des urgences ${ }^{9}, \mathrm{M}$. Palmer consacre désormais une vingtaine d'heures par semaine à l'Association des Médecins du Massachusetts (Massachusetts Medical Society). Quant à R. Cook, il se consacre totalement à l'écriture, mais reste affilié au Massachusetts Eye and Ear Infimaryde Boston et se dit prêt à reprendre ses activités de neurochirurgien si nécessaire.

9 T. Gerritsen, médecin généraliste de formation, a abandonné assez tôt la pratique de la médecine pour pouvoir élever ses enfants tout en se livrant à sa passion, l'écriture. Toutefois, sa connaissance intime du milieu médical ne fait pas de doute. La journaliste Y. Daley confirme cette compétence dans un article électronique sur le site du Stanford Magazine, intitulé "Pulse Fiction »: "Like lawyer-writers John Grisham and Scott Turow, Gerritsen uses inside knowledge of an insular profession to lend her books authenticity. 》 (2003)

Le deuxième critère emblématique, celui de la représentativité professionnelle, nous semble donc validé. Ainsi, la reconnaissance de R. Cook par la communauté médicale estelle illustrée par le fait que deux médecins lui ont demandé d'écrire la préface de leur 
ouvrage spécialisé Xeno: The Promise of Transplanting Animal Organs Into Humans (Cooper \& Lanza : 2000).

41 L'appellation de "thrillers» spécialisés s'applique également aux textes fondateurs, qui sont caractérisés par une intrigue à rebondissements, une forte dose de suspense et l'exacerbation des émotions du lecteur. Nous verrons toutefois plus loin que les auteurs font parfois des infidélités au genre du roman à suspense stricto sensu. C'est le cas de R. Cook (Invasion) et de T.Gerritsen (Gravity), qui ont emprunté des éléments emblématiques au genre de la science-fiction pour diversifier leurs productions.

Enfin, les critères essentiels du contenu médical narratif et discursif sont également présents dans les romans de R. Cook, M. Palmer et T. Gerritsen. Nous avons eu l'occasion de présenter la prédominance du substrat professionnel dans leurs productions ${ }^{10} \mathrm{et} \mathrm{ici}$, nous nous contenterons de citer ce commentaire de B. Pomidor qui, dans un entretien avec la journaliste B. A. Gabriel, souligne la parfaite compatibilité entre forme narrative et contenu discursif professionnel au cœur de la FASP médicale :

"There is a real parallel between mysteries and medicine," says Dr. Pomidor,

"between the solving of a murder mystery and the clinical problem-solving process.

So it didn't seem like too much of a stress for a pair of doctors to use their medical

skills to solve mysteries." (2002)

\subsection{Les prototextes}

43 F. G. Slaughter et M. Crichton sont les principaux inspirateurs de la fiction médicale spécialisée fondée sur l'expérience professionnelle des auteurs. Il est donc pertinent de vérifier si certains de leurs romans méritent d'être inclus dans la sphère des textes fondateurs de la FASP médicale.

Nous avons choisi de qualifier leurs productions à forte influence médicale de « prototextes » car elles ont fait leur apparition dans une période précédant l'apparition des romans à suspense à dominante médicale. Dans le continuum de la création littéraire, elles s'insèrent entre les genres établis et le genre récemment défini de la FASP.

Il est avéré que les romans de F. G. Slaughter et de M. Crichton sont des productions commerciales de masse. Au lendemain de la Seconde Guerre mondiale, F. G. Slaughter était un auteur extrêmement populaire. Aujourd'hui encore, les « technothrillers » produits par M. Crichton connaissent un succès international.

En revanche, leurs productions n'entrent pas dans le cadre strict des romans contemporains à visée commerciale. Si le dernier ouvrage de F. G. Slaughter, Transplant, a été publié en 1987, la majeure partie de son œuvre remonte aux années quarante, cinquante et soixante.

47 M. Crichton a été l'un des premiers écrivains à utiliser le substrat professionnel médical de façon homogène dans des romans à suspense comme A Case of Need (1968) et The Terminal Man (1972). Toutefois, il a délaissé l'écriture de medical thrillers au début des années soixante-dix pour se consacrer à l'écriture de "technothrillers", autre genre spécialisé de la FASP dont il est devenu l'une des figures emblématiques.

Pour ce qui est du critère professionnel, les deux auteurs connaissaient le milieu de la médecine de l'intérieur. Avant de se consacrer à l'écriture, F. G. Slaughter fut un chirurgien réputé en Floride. M. Crichton a fait des études de médecine en bonne et due 
forme. Il a même écrit un livre Five Patients: The Hospital Explained (1970) qui témoigne avec réalisme de son expérience en tant qu'interne. romans médicaux de F. G. Slaughter au cœur de la FASP médicale. Il nous semble qu'il serait plus judicieux de les classer dans la catégorie "The Period FASP» définie par S. Isani : « However, in spite of these apparent shortcomings, to dismiss the period FASP as a minor anachronism of the genre would be to underestimate its importance in the evolution of the genre. " (2004:31) doute préparé le terrain qui allait favoriser le développement ultérieur de la FASP médicale. M. Crichton, lui, a joué un rôle plus central. Ses premiers romans (A Case of Need et The Terminal Man) possèdent les caractéristiques génériques principales des medical thrillers. À ce titre, ils méritent d'être qualifiés de véritables prototextes au sein de la FASP médicale puisque des écrivains comme T. Gerritsen reconnaissent l'influence exercée par leur auteur:

As far as which particular writers have inspired me, some of my favorites include Stephen King, whose horror novels have always impressed me because of his acute observations of human behavior, and also Michael Crichton, because of his creative use of science in his stories. (Gerritsen InCharpy, 2004b)

\section{Aux marges de la FASP médicale}

Après avoir parcouru l'aire des textes généralement répertoriés au cœur de la FASP médicale, nous allons maintenant explorer les vastes frontières du genre. Nous appellerons les romans appartenant à cette zone limitrophe large «textes périphériques » car leur repérage devrait nous permettre de mieux délimiter les contours du genre. 
55 Le positionnement des productions gravitant à proximité du noyau dur des textes fondateurs est délicat à établir, car elles répondent à plusieurs critères emblématiques de la FASP médicale et de nombreuses zones de chevauchement existent entre le cœur et la périphérie. En effet, ces romans commerciaux contemporains comportent fréquemment des éléments de suspense et un discours médical, paramédical ou biomédical affirmé. Néanmoins, la formation professionnelle de leurs auteurs est parfois atypique et le substrat (ou l'adstrat) professionnel contextuel et discursif qui les caractérise ne relève pas toujours exclusivement du domaine strictement médical.

$\mathrm{Au}$ cours de notre exploration, nous distinguerons les textes périphériques à tendance autobiographique (J. Connelly), dans lesquels le vécu de l'auteur nourrit la narration et le discours, des textes périphériques protéiformes ${ }^{11}$ (G. Braver, K. Reichs), alimentés par plusieurs genres existants.

\subsection{Les textes périphériques à tendance autobiographique}

J. Connelly est un auteur quelque peu difficile à classer. Son premier roman Bringing Out the Dead (1998) puise grandement dans son expérience en tant qu'ambulancier dans le quartier new-yorkais de «Hell's Kitchen ». La richesse autobiographique et l'atmosphère urbaine très noire du livre ont attiré l'attention du réalisateur $\mathrm{M}$. Scorsese, qui en a fait une adaptation cinématographique dès 1999.

i J. Connelly avait comme objectif de commencer des études médicales après avoir obtenu son diplôme d'ambulancier en 1986, il se tourna rapidement vers l'écriture. Cependant, son intérêt précoce pour la médecine et ses connaissances dans le domaine paramédical constituent un socle professionnel solide sur lequel Bringing Out the Dead repose en partie.

59 Ce premier roman a connu un succès commercial marqué, qu'il doit davantage à ses personnages hauts en couleur et à son style flamboyant qu'à une intrigue haletante. Il n'est donc pas possible de dire que Bringing Out the Dead est un thriller spécialisé.

Toutefois, force est de constater que la course contre le temps livrée par les ambulanciers et le contexte mouvementé de la médecine urgentiste jouent sur les ressorts de la temporalité, qui est un des traits essentiels des romans à suspense classiques. Ce commentaire du New York Post, extrait du paratexte éditorial de Bringing Out the Dead, souligne ce point :

With nine years' experience as a paramedic, Connelly is in full control of this material [...] Bringing Out the Dead is fused with tension and fear, and its vivid descriptions of OD's and emergency pregnancies keep the pages turning. (1998: ii)

61 Le roman de J. Connelly est empreint d'un substrat professionnel médical ou paramédical réaliste, tant sur le plan narratif que discursif. La scène suivante, tirée de Bringing Out the Dead, pourrait fort bien provenir d'un des medical thrillers produits par M. Palmer :

"Come here," Hazmat said, and pulled my arm. He pointed to a middle-aged man lying in unit six. "I've got a first-time heart attack here, forty-five years old. He should have gone to the CCU ten hours ago. He needs catherization, venous central pressure monitoring, EKGs every thirty minutes. I couldn't do it here even if I had the time, but the CCU's full. There's three bodies up there like the one you just brought in." (1998: 40)

Avec M. Petit, nous dirons donc que «si les romans de la FASP sont généralement ou habituellement des thrillers, ils ne le sont pas nécessairement.» (2004: 6). À ce titre, 
Bringing Out the Dead, qui n'est pas un thriller spécialisé, semble appartenir à une zone périphérique du genre de la FASP médicale. Il s'agit d'un roman à substrat professionnel dense qui, sans être un medical thriller classique, éveille chez le lecteur des émotions liées à une charge autobiographique forte.

\subsection{Les textes périphériques protéiformes} propose des cours sur le roman policier, le roman de science-fiction et le roman d'épouvante, tout en animant des ateliers d'écriture. Depuis le début des années quatrevingt-dix, parallèlement à ses activités universitaires, il s'est lancé dans la rédaction de romans à visée commerciale qu'il qualifie lui-même de "Medical or Biomedical Suspense » (2003).

À la demande de son éditeur, il a adopté le nom de plume G. Braver en vue de la publication de son quatrième roman Elixir (2000), version biomédicale moderne du Portrait of Dorian Gray d'O. Wilde. Si l'auteur traite de questions de société à dominante médicale ou biomédicale dans ses romans, il n'appartient pas au milieu professionnel de la santé.

Bien qu'il ait eu une solide formation universitaire de physicien et qu'il s'intéresse de près aux problèmes éthiques posés par les découvertes scientifiques, il n'a pas une connaissance intime du monde de la médecine. Son positionnement au sein de la FASP médicale est donc problématique.

En revanche, il nous semble que G. Braver est à sa place dans le "medical suspense genre » au sens large du terme. Ses productions sont des textes périphériques proches du noyau central de la FASP médicale. En effet, il a de fréquents contacts électroniques avec T. Gerritsen dont il utilise régulièrement le premier medical thriller, Harvest, avec ses étudiants. Il dit également être un avide lecteur des romans à suspense de R. Cook.

D'une part, ses romans sont caractérisés par une intrigue à rebondissements ; des gens ordinaires, pas nécessairement issus du milieu médical ou scientifique, y mènent l'enquête afin de résoudre une énigme (bio)médicale. D'autre part, le substrat (en fait, souvent, l'adstrat) professionnel présent dans ses romans est à forte influence scientifique, tantôt à dominante médicale, tantôt à tendance biomédicale ou biologique, comme le montre cet extrait de Gray Matter :

"For obvious reasons, I cannot tell you who they are," he said. "But working with me will be the best there is, I assure you."

"Practicing neurosurgeons?"

"Practicing neurosurgeons, anaesthesiologists, scrub nurses, circulation nurses the full complement."

Rachel nodded, questions jamming her mind. "The other day you mentioned the cocktail of ingredients you'd be using," Rachel began, unable to actually say the words injecting into my son's brain.

"Yes. I won't bore you with details, but the whole field of implantation is very intricate and complicated. But we use a mixture of certain chemical stimulants, protein growth factors, and dissociated tissue cells which will on their own genetic program create new axonal structures where deficient." (2004: 281)

Il convient également de noter que G. Braver a recours à plusieurs genres (science-fiction, épouvante, "technothrillers»), ce qui fait de lui "the master of medical techno-horror " (2002). Cet autre passage de Gray Matter l'illustre également : 
Rachel moved closer to the child banded with green. It was Dylan. Rachel groaned. He was breathing through a respirator. His head had been shaved and slathered with some bitter-smelling jelly-stuff - like burnt almonds. Clamped to his skull was a metal frame that looked like some medieval torture device. No probes had yet been inserted into his head, but a high-speed drill was poised to bore its way through his skull above his left ear. On the stainless steel tray beside Dylan's head lay surgical knives, drill bits, and other glistening steel tools. (2004 : 458-459)

Pour toutes ces raisons, G. Braver est un auteur atypique dont les romans protéiformes à influence scientifique ou biomédicale se situent souvent aux confins de la FASP médicale. En revanche, les romans de K. Reichs nous semblent beaucoup plus proches du cœur de la FASP médicale car ils possèdent pratiquement tous les critères emblématiques du genre spécialisé.

Depuis la publication de Déjà Dead (1997), l'auteur n'a cessé d'accumuler les succès de librairie, de hall de gare ou d'aéroport. Ses romans appartiennent bien à la catégorie de la fiction commerciale contemporaine dont K. Reichs est rapidement devenue l'une des figures de proue. Ses romans sont tellement populaires que la chaîne américaine 20th Century Fox Television lui a proposé l'adaptation au petit écran des aventures de son héroïne fétiche, Temperance Brennan ${ }^{12}$.

Du point de vue de l'appartenance au milieu professionnel, K. Reichs n'a rien à envier aux auteurs centraux de la FASP médicale. Son statut de professionnelle de l'anthropologie médico-légale est bien établi; elle travaille à la fois pour les services centraux de médecine légale de l'État de Caroline du Nord et le Laboratoire des sciences judiciaires et de médecine légale de la province du Québec. En outre, son expertise en tant qu'anthropologue au service de la médecine légale lui a valu de témoigner dans de nombreux procès internationaux, en particulier devant la Cour de justice des NationsUnies au sujet du génocide rwandais.

Certes, les romans de K. Reichs ne sont généralement pas classés par les éditeurs dans la catégorie des medical thrillers, mais dans celle des "forensic thrillers ", au confluent de la FASP médicale et de la FASP juridique. Néanmoins, son impact sur la communauté professionnelle est bien plus large que ne le laisse entendre cette appellation quelque peu réductrice.

La consultation de son site officiel ${ }^{13}$ permet de constater qu'elle donne de nombreuses conférences devant des associations de spécialistes de la médecine comme The American College of Orthopedic Surgeons ou The Quebec Association of Oncologists and Hematologists.

Pour ce qui est de l'appartenance au genre du thriller spécialisé, il apparaît que l'auteur sait mener une enquête - qu'elle relève du domaine privé ou de la police - tout en ménageant les effets de suspense. Le paratexte éditorial du journaliste de People au sujet de Death du Jour confirme cette compétence : « Atmospheric, suspensefully paced ... Likely to leave you with the shivers. » (2000 : iii)

75 Tout comme G. Braver, K. Reichs a recours à des emprunts aux genres littéraires du roman policier ou du roman d'épouvante, afin de créer une atmosphère propice au suspense. Ses forensic thrillers sont à la fois spécialisés d'un point de vue professionnel et hybrides d'un point de vue littéraire.

Enfin, le dernier critère emblématique retenu, à savoir celui du substrat professionnel, imprègne les romans de K. Reichs. Cet extrait technique de Death du Jour montre que 
l'auteur exploite pleinement sa connaissance du discours de l'anthropologie médicolégale :

Wound A is a circular defect, of which only the superior half is preserved. It is localized to the midfrontal region, lying approximately 2 centimeters above glabella and 1.2 centimeters to the left of midline. The defect measures 1.4 centimeters in diameter and presents characteristic bevelling of the inner table. Charring is present along the margins of the defect. Wound A is consistent with a gunshot entrance wound. (2000:60-61)

Tous ces éléments convergents devraient permettre de placer K. Reichs au centre de la FASP médicale. Toutefois, son attachement au substrat professionnel de l'anthropologie médico-légale la place plutôt dans une zone périphérique proche, souvent fusionnelle. Comme nous allons le voir maintenant, l'influence de ces romans protéiformes et leur interaction avec les textes centraux du genre spécialisé de la FASP médicale sont loin d'être négligeables.

\section{4. Évolution du genre spécialisé : The Sinner de T. Gerritsen}

La représentation circulaire que nous avons donnée de la FASP médicale, avec un cœur et des marges, est utile car elle permet de positionner les romans en fonction des forces centrifuges et centripètes en œuvre au sein du genre.

79 Toutefois, ce mode de représentation est quelque peu frustrant, parce que binaire. En effet, il ne permet pas de faire apparaître les zones de fusion, qui sont un des signes de l'interpénétration et de l'évolution des genres. J-M. Schaeffer propose un commentaire intéressant sur ce phénomène proche de l'osmose :

Quant à la question de la spécificité éventuelle des noms génériques liés à la littérature, elle ne saurait être résolue d'emblée, contrairement à ce que présupposent toutes les classifications systématiques des genres littéraires. Cela est dû notamment au fait que la littérature n'est pas une notion immuable: si nous partons de l'acception actuelle du texte (et de quelle autre pourrions-nous partir ?), nous sommes obligés de convenir qu'il s'agit, non pas d'une classe unique de textes fondée sur des critères constants, mais d'un agrégat de classes fondées sur des notions diverses, ces classes étant le précipité actuel de toute une série de réaménagements historiques obéissant à des stratégies et des critères notionnels divers. (1989: 76)

80 Les genres - qu'ils soient littéraires ou spécialisés - sont souvent considérés comme des formes figées car ils sont définis par des traits généraux conventionnels. Comme il existe des zones de chevauchement entre les genres, il est parfois difficile de les répertorier. En réalité, les genres ont une vie dynamique et leurs conventions évoluent en fonction de facteurs économiques, sociaux et idéologiques, en particulier dans le domaine de la fiction commerciale. Cette évolution produit fréquemment des groupes génériques en mutation ou des genres hybrides.

81 Dans une collection consacrée à la théorie de la littérature dirigée par T. Todorov, le formaliste russe B. Tomachevski fournissait déjà un éclairage pertinent sur ce point :

Le genre s'enrichit d'œuvres nouvelles qui se rattachent aux œuvres déjà existantes du genre donné. La cause qui a promu un genre peut ne plus agir; les traits fondamentaux du genre peuvent lentement changer, mais le genre continue à vivre en tant qu'espèce, c'est-à-dire par le rattachement habituel des œuvres nouvelles aux genres déjà existants. $(1965: 303)$ 

En effet, le roman récemment publié de T. Gerritsen, The Sinner,présente des traits qui le rattachent à la fois à la catégorie des medical thrillers et à ce que S. Isani appelle «the Panachée FASP 》 (2004 : 28).

Comme nous l'avons indiqué précédemment, T.Gerritsen est un des auteurs emblématiques de la FASP médicale. Elle a assis sa réputation avec The Surgeon (2001) et sa suite, The Apprentice (2002), dont certaines scènes rappellent l'atmosphère angoissante des « gothic novels » de la période pré-romantique.

Nous avons également constaté, en faisant référence à Gravity, que l'auteur ne se limitait pas au genre spécialisé du roman à suspense à dominante médicale, mais qu'elle avait déjà emprunté des éléments à d'autres genres littéraires - en l'occurrence celui du roman de science-fiction.

The Sinner présente toutes les caractéristiques emblématiques de la FASP médicale. Ainsi le contexte narratif et le contenu discursif de cette scène particulièrement réaliste, située au service de réanimation, sont-ils dignes des meilleurs romans à suspense à dominante médicale de M. Palmer :

"PVCs," the nurse said. "I'm seeing PVCs!"

Gazes shot straight to the cardiac monitor. The heart tracing, which had been

ticking rapidly but evenly across the screen, was now distorted by dagger thrusts.

"V tach!" said Yuen.

"I can't get any pressure! She's not perfusing."

"Get that bed rail down. Come on, come on, let's start compressions." (2004:249)

T. Gerritsen met sa connaissance personnelle de la médecine au service du substrat professionnel médical et elle y ajoute un volet original avec l'ajout d'adstrat professionnel lié à la présence du médecin légiste Maura Isles, qui partage désormais le devant de la scène aux côtés de l'héroïne des romans précédents, le détective Jane Rizzoli :

The darkly mottled wall of the right ventricle, and the white apical scar, told her this heart's sad story. An old myocardial infarction, months or even years old, had already destroyed part of the left ventricular wall. Then, sometime in the last twenty-four hours, a fresh infarction had occurred. A thrombus had blocked off the right coronary artery, strangling the flow of blood to the muscle of the right ventricle.

She resected tissue for histology, already knowing what she would see under the microscope. Coagulation and necrosis. The invasion of white cells, moving in like a defending army. (2004: 8)

T. Gerritsen n'est d'ailleurs pas le seul auteur de la FASP médicale à combiner des scènes de médecine traditionnelle et des scènes de médecine légale. Le personnage central des romans de L. Goldberg, le docteur Joanna Blalock, est une pathologiste spécialisée dans la médecine légale.

Cette alternance récente de "substrat professionnel» médical et "d'adstrat professionnel $\aleph^{14}$ médico-légal indique que la FASP médicale semble évoluer vers des formes mixtes, caractérisées par le mélange subtil de traits classiques et de traits empruntés à d'autres genres. À ce sujet, le commentaire d'A. Tudor au sujet de la mutation des genres cinématographiques pourrait fort bien être appliqué à The Sinner :

First, in that innovations are added to an existing corpus rather than replacing redundant elements, it is cumulative. Second, in that these innovations must be basically consistent with what is already present, it is "conservative". (1974 : 225-226) 


\section{Conclusion}

Dans cette étude, nous avons rappelé les fondements théoriques de la fiction à substrat professionnel et nous avons utilisé les critères emblématiques de la FASP définis par M. Petit pour répertorier les principaux auteurs et quelques romans du genre spécialisé de la FASP médicale. En nous appuyant sur cette nomenclature stricte, nous avons isolé un noyau dur d'auteurs contemporains (R. Cook, M. Palmer, T Gerritsen) dont les medical thrillers comportent la totalité des traits distinctifs du genre.

En amont, nous avons repéré quelques auteurs (F. G. Slaughter, M. Crichton), dont les romans à dominante médicale ont pu servir de prototextes dans une période précédant l'essor de la FASP médicale. À ce titre, ils peuvent être considérés comme précurseurs.

En aval, nous avons répertorié plusieurs romanciers présumés atypiques (J. Connelly, G. Braver, K. Reichs) dont les productions commerciales contemporaines sont pourtant souvent présentées par les éditeurs et classées par les libraires comme des medical thrillers . Comme leurs romans ne répondent pas à tous les critères emblématiques du genre, nous les avons placés à la périphérie du domaine de la FASP médicale sous le label « textes périphériques ».

Cette approche normative nous a permis de définir les lignes de force et les points névralgiques du genre spécialisé. Néanmoins, elle ne fournit qu'une vision globale du domaine générique, dont les frontières parfois diffuses ont besoin d'être observées de plus près. Comme l'indique J.-M. Schaeffer :

Après tout, ce sont les genres littéraires qui m'intéressent. Simplement, je plaide pour des frontières floues et mouvantes et je m'accorde le droit de les traverser ou de les ignorer de temps en temps. (1989: 77)

Certes, les auteurs des textes situés au cœur du genre, mais aussi la plupart de ceux qui sont censés être à la marge, s'appuient sur leur connaissance ou leur expérience technique, linguistique et culturelle du milieu professionnel. Nous pouvons donc affirmer que le substrat professionnel spécialisé constitue l'épine dorsale de la FASP médicale.

4 Toutefois, certaines zones du domaine sont beaucoup plus denses et diversifiées. Elles révèlent l'alternance de traits distinctifs de plusieurs genres et l'apparition d'un processus récent qui s'apparente à l'osmose. Si l'on se réfère à l'étymologie de ce mot (en grec ôsmos signifie "poussée, impulsion»),c'est ce principe d'interpénétration et de dilution sélective qui est susceptible de donner un nouvel essor au genre.

Selon les principes de la transmutation des genres, plusieurs auteurs en quête de renouvellement s'inspirent des «pré-textes » de genres littéraires existants ou d'autres genres spécialisés pour étayer le substrat professionnel médical qui sous-tend et fédère leurs romans.

Les références au genre de la science-fiction dans Invasion de R. Cook et les emprunts fréquents au genre spécialisé du forensic thriller dans The Sinner de T. Gerritsen sont des indications objectives de l'évolution de la FASP médicale. Après avoir épuisé les thèmes centraux du genre spécialisé, les écrivains de la FASP médicale se livrent à des variations génériques pour maintenir l'intérêt de leurs lecteurs habituels et sans doute aussi, pour attirer de nouveaux lecteurs dans un contexte de concurrence éditoriale féroce.

Sans délaisser totalement les critères fondamentaux du roman à suspense à dominante médicale, les auteurs des textes fondateurs centraux absorbent progressivement des 
éléments constitutifs de genres à fort impact commercial (la science-fiction) ou de genres connexes à succès (le thriller médico-légal).

Nous laisserons le mot de la fin à G. Braver qui, dans la partie «Author Profile »du site électronique Bookreporter (2002),propose un commentaire résumant assez bien l'évolution du genre spécialisé et les limites que se fixent les auteurs de ce type de fiction commerciale : «There are no new myths, no new story paradigms... only new twists. What I try to do is to invent variations of old allegories. It's the most any writer can strive for ».

\section{BIBLIOGRAPHIE}

\section{Références du corpus}

Braver, G. 2001 [2000]. Elixir. New York : Tor.

Braver, G. 2004 [2002]. Gray Matter. New York : Tor.

Connelly, J. 1999 [1998]. Bringing Out the Dead. New York : Vintage Contemporaries.

Cook, R. 1978 [1977]. Coma. Londres : Pan Books.

Cook, R. 1997. Invasion. New York : Berkley Books.

Cooper, D.K.C. \& R.P. Lanza. 2000. Xeno: The Promise of Transplanting Animal Organs Into Humans. New York : Oxford University Press.

Crichton, M. (sous le nom de Jeffery Hudson). 1969 [1968]. A Case of Need. New York : Signet.

Crichton, M. 1970. Five Patients: The Hospital Explained. New York : Knopf.

Crichton, M. 2002 [1972]. The Terminal Man. New York : Avon Books.

Crichton, M. 1999 [1978]. Coma (DVD). Burbank : Turner Entertainment Co. and Warner Home Video.

Gerritsen, T. 1997 [1996]. Harvest. New York : Pocket Star Books.

Gerritsen, T. 2002 [2001]. The Surgeon. New York :Ballantine Books.

Gerritsen, T. 2003 [2002]. The Apprentice. New York :Ballantine Books.

Gerritsen, T. 2004 [2003]. The Sinner. New York :Ballantine Books.

Goldberg, L. 2003, Fever Cell. New York : Signet.

Palmer, M. 1995 [1982]. The Sisterhood. New York : Bantam Books.

Palmer, M. 1997 [1996]. Critical Judgement. Londres : Arrow.

Pears, I. 2001 [1992]. The Bernini Bust. New York : Berkley Books.

Reichs, K. 1998 [1997]. Déjà Dead. New York : Pocket Books.

Reichs, K. 2000 [1999]. Death du Jour. New York : Pocket Books.

Scorsese, M. 1999. Bringing Out the Dead (DVD). New York : Touchstone Pictures. 
Slaughter, F. G. 1983. Doctors at Risk. Londres : Hutchinson.

\section{Références}

Birken, G.2005.« Medical fiction writing: Breaking through. Preconference: Friday, October 21, 2005 ». Sea Crest Oceanfront Resort, Falmouth, Cape Cod, MA. 7 juillet $2005<$ http:// www.seak.com>.

Bookreporter. 2002. 7 juillet 2005 <http://www.bookreporter.com/authors/au-braver-gary.asp>. Chandler, D. 2003. Introduction to Genre Theory. Aber. 07 juillet 2005. <http://www.aber.ac.uk/ media/Documents/intgenre/intgenre1.html>.

Charpy, J-P. 2004a. « Le roman à suspense à dominante médicale : typologie d'un sous-groupe générique ». In Petit, M. et S. Isani. Aspects de la fiction à substrat professionnel. Bordeaux : Université Victor Segalen Bordeaux 2, collection Travaux 2025, 65-82.

Charpy, J.-P. 2004b. « Milieux professionnels et FASP médicale : de l'autre côté du miroir ». ASp 45-46, 61-79.

Diaz-Santos, G. 2000. « Technothrillers and English for science and technology ». English for Specific Purposes 19, 221-236.

Gabriel, B. 2002.«Physician novelists: At the intersection of writing and healing ». <http:// www.aamc.org/newsroom/reporter/nov02/physician.htm>.

Goshgarian, G. 2003.« Gary Braver, an interview by Kilian Melloy ». <http://www. infinityplus.co.uk/ nonfiction/intbraver.htm>.

Harkavy, J. 2002. « Authors Train Fellow Doctors to Pen Fiction Novels ». The Columbia Tribune, 29 décembre 2002. <http://www.archive.columbiatribune. com>.

Isani, S. 2004. « The FASP and the Genres within the Genre ». In Petit, M. et S. Isani. Aspects de la fiction à substrat professionnel. Bordeaux : Université Victor Segalen Bordeaux 2, collection Travaux 2025, 25-36.

Petit, M. 1999. « La fiction à substrat professionnel : une autre voie d'accès à l'anglais de spécialité ». ASp, 23-26,57-81.

Petit, M. 2000. « Le paratexte dans la fiction à substrat professionnel ». Bulletin de la société de stylistique anglaise 21, Texte et paratexte, Actes du Colloque de Nanterre 4-5 juin 1999, 173-195.

Petit, M. 2004. «Quelques réflexions sur la fiction à substrat professionnel : du général au particulier ». In Petit, M. et S. Isani. Aspects de la fiction à substrat professionnel. Bordeaux : Université Victor Segalen Bordeaux 2, collection Travaux 2025, 3-23.

Physician's Weekly. 2000. 31 juillet. Vol. 17/29 <http://www.physiciansweekly.com>.

Schaeffer, J.-M. 1989. Qu'est-ce qu'un genre littéraire? Paris : Seuil.

Todorov, T. (dir.). 1965.Théorie de la littérature. Textes des formalistes russes. Paris : Seuil.

Tudor, A. 1974. Image and Influences: Studies in the Sociology of Film. Londres : George Allen \& Unwin.

\section{NOTES}

1. Ces travaux portent sur la typologie du groupe générique spécialisé de la FASP médicale (Charpy 2004a) et sur les relations entre le milieu professionnel de la médecine et la FASP médicale (Charpy 2004b). 
2. Voir supra, introduction.

3. Les productions de la FASP médicale télévisuelle (E.R., à l'origine écrit et co-produit par M. Crichton) ou cinématographique (l'adaptation par M. Crichton du roman de R. Cook Coma ou celle du roman de J. Connelly Bringing Out The Dead par M. Scorsese) restent minoritaires et, dans le cas de la FASP cinématographique, beaucoup moins populaires que les best-sellers initiaux.

4. Voir Charpy (2004a : 75).

5. Contact électronique avec T. Gerritsen en date du 19 avril 2004.

6. Voir < http://www.bma.org.uk>.

7. Voir supra, section 1.1.

8. Avant la publication de son premier roman à suspense à dominante médicale Harvest (1996),

T. Gerritsen s'était spécialisée dans la rédaction de romans sentimentaux comme Call After Midnight (1986). Elle fait partie de la deuxième génération d'auteurs de la FASP médicale, mais la cohérence générique de ses productions récentes permet de les classer parmi les textes fondateurs.

9. Voir Critical Judgement (1997 : 7-12, 21-33, 59-67, 75-78, 122-137, 205-215, 226-227, 301-303).

10. Voir Charpy (2004: 72-75).

11. Voir la notion de "The hybrid FASP" (Isani $2004: 30$ ).

12. La série télévisée, intitulée Bones, devrait être diffusée aux heures de grande écoute à l'automne 2005.

13. Voir <http://www.kathyreichs.com>.

14. Dans la partie Remerciements du roman, T. Gerrisen cite clairement ses sources : «My warmest thanks to [...] Margaret Greenwald, M.D., for allowing me a look into the medical examiner's world. » (2004 : vii).

\section{RÉSUMÉS}

Le but de cet article est d'analyser divers textes de la FASP médicale en tant qu'émanation du genre de la fiction à substrat professionnel (FASP). Il est convenu de dire que les romans d'un petit groupe d'écrivains américains (R. Cook, M. Palmer, T. Gerritsen) forment le noyau dur de la FASP médicale. L'auteur se penche sur les prototextes et les textes périphériques écrits par des romanciers (F.G. Slaughter, M. Crichton, d'une part; J. Connelly, G. Braver, K. Reichs, d'autre part) réputés être aux marges du genre. Après avoir étudié les liens complexes entre la nature protéiforme de ces textes et les normes du genre, l'auteur émet l'hypothèse que la FASP médicale évolue graduellement par osmose. Il s'appuie sur le roman de T. Gerritsen The Sinner (2003) afin de montrer que les écrivains de la FASP médicale s'inspirent de plusieurs genres et pré-textes existants dont ils se servent comme cadre pour étayer le substrat professionnel de leurs romans.

The purpose of this article is to analyse various medical thrillers belonging to the genre of professionally-based fiction. It is generally agreed that the novels of a small group of American writers (R. Cook, M. Palmer, T. Gerritsen) form the core of professionally-based medical fiction. The author looks into the prototexts and the peripheral texts of novelists - F.G. Slaughter and M. Crichton on the one hand; J. Connelly, G. Braver, K. Reichs on the other hand - said to be on the fringes of the genre. After studying the complex relationships between the protean nature of these texts and the standards of the genre, the author postulates that professionally-based medical fiction is gradually evolving by osmosis. He uses T. Gerritsen's novel The Sinner (2003) to 
show that writers of professionally-based medical fiction draw their inspiration from several existing genres and pre-texts, which they use as a framework to prop up professionally-based elements in their novels.

\section{INDEX}

Mots-clés : FASP, FASP médicale, genre, prototexte, texte périphérique

Keywords : peripheral text, professionally-based fiction, professionally-based medical fiction, prototext

\section{AUTEUR}

\section{JEAN-PIERRE CHARPY}

Jean-Pierre Charpy est professeur agrégé d'anglais hors-classe à la faculté de médecine de Dijon. Il a soutenu une thèse intitulée « Médias, médium et modalité » en 1998 à l'université de Bourgogne. Il est le co-auteur du guide pratique (Ellipses, 2002) et du CD-ROM « La communication orale scientifique en anglais » (Edulang, 2003). Ses domaines de recherche portent sur la médiologie, le discours scientifique et la FASP médicale. jean-pierre.charpy@ubourgogne.fr 